\title{
Rentabilidad del Trabajo en Chile: Análisis de la Evolución de los Retornos por Nivel Educativo
}

\section{PERFORMANCE OF THE WORK IN CHILE: ANALYSIS OF THE EVOLUTION OF RETURNS BY EDUCATION LEVEL}

\author{
Guillermo Riquelme Silva', Víctor E. Olivares-Faúndez² \\ 1. Centro de Estudios y Gestión Social, Universidad Autónoma de Chile, Chile. \\ 2. Escuela de Psicología, Universidad de Santiago de Chile, Chile.
}

\begin{abstract}
RESUMEN
La presente investigación tiene como propósito realizar un análisis econométrico de la rentabilidad del trabajo calificado en Chile, tanto a nivel general como para sus distintos niveles formativos, con especial referencia en el nivel de educación superior. El análisis de los datos se efectuó considerando la clásica ecuación de ingresos de Mincer, adaptada a través del método spline para determinar las rentabilidades de cada nivel formativo. Se trabaja con datos de panel de corte transversal incluyendo los años 1992, 2000, 2009 y 2011, obtenidos de la Encuesta de Caracterización Socioeconómica Nacional (CASEN). Los resultados destacan que el nivel de educación superior en Chile es el sector más rentable de los distintos niveles educativos, considerando todos los años estudiados.
\end{abstract}

(Riquelme G, Olivares V, 2015. Rentabilidad del Trabajo en Chile: Análisis de la Evolución de los Retornos por Nivel Educativo. Cienc Trab. Ene-Abr; 17 [52]: 69-76).

Palabras Clave: TRABAJO CALIFICADO, RENTABILIDAD EDUCACIONAL, CAPITAL HUMANO, ECONOMIIA DE LA EDUCACIÓN.

\section{ABSTRACT}

This research deals with the econometric analysis of the profitability of qualified Chile, both as to its various educational levels generally, with special reference to higher education level work. As a methodology, classical Mincer earnings equation, adapted through the spline method for determining the profitability of each training level is used. Working with panel data from cross section for the years 1992, 2000, 2009 and 2011, obtained from the Encuesta de Caracterización Socioeconómica Nacional (CASEN). As resulting emphasize that higher education in Chile is the most profitable of the different educational levels for all years studied.

Key words: WORKING QUALIFIED PERFORMANCE EDUCATION, HUMAN CAPITAL, ECONOMICS OF EDUCATION.

\section{INTRODUCCION}

Según la teoría del Capital Humano ${ }^{1}$, el aumento de la educación de las personas posibilitaría también un aumento de sus respectivos niveles de ingresos. En tal sentido, la educación puede ser considerada como inversión y, por consiguiente, se espera de ella ciertos retornos en el tiempo. La relación entre la inversión y los retornos de la educación configurarían dentro de la literatura económica de la educación la denominada Rentabilidad de la Educación. ${ }^{2}$

La rentabilidad de la educación pasa a convertirse en un indicador relevante para la toma de decisiones de los agentes económicos, donde los acuerdos de inversión en esta materia cobran real relevancia dentro de las políticas públicas. Específicamente se alude al

\section{Correspondencia / Correspondence:}

Centro de Estudios y Gestión Social del Maule,

Universidad Autónoma de Chile, Sede Talca.

5 poniente 1670, Edificio Aulas 5, Primer Piso. Talca, Chile.

Tel.: (+56) 712735690.

e-mail: griquelmes@uautonoma.cl,•Página web: www.cegestionsocial.cl

Recibido: 12 de Enero 2015 / Aceptado: 09 de Marzo
Estado y su rol como actor económico en la educación nacional. Cabe recordar que el estado debe hacer un uso eficaz y eficiente de los recursos públicos y definir prioridades para todas y cada una de sus áreas de inversión social, en particular, en lo que respecta al presente estudio, la educación. Asimismo, debe evaluar los proyectos de inversión social para direccionar los recursos de la manera más eficiente, dadas las prioridades existentes. En tal sentido, la rentabilidad de los recursos invertidos en educación y en cada uno de sus niveles por el estado es fundamental. ${ }^{3}$

La pregunta que surge entonces es: ¿Cuál es la rentabilidad de la educación? La respuesta a esta pregunta no parece, a priori, fácil de contestar dadas las características intangibles de la educación y su volatilidad en el tiempo. No obstante, la literatura económica en educación ha sido bastante fructífera teniendo sus primeros aportes a comienzos de la segunda mitad del siglo pasado, cuando surgen importantes trabajos teóricos, siendo los más destacados aquellos focalizados en la determinación de ecuaciones de ingresos, como los de Mincer, Ben Porath, Willis y Heckman et al. ${ }^{4-8}$

Por su parte, en Chile, se destacan los desarrollados por Beyer ${ }^{9-11} \mathrm{y}$ Sapelli ${ }^{12-13}$, quienes adaptan y aplican las ecuaciones de Mincer en la realidad educativa nacional (aspectos que serán analizados más adelante en este trabajo). Cabe destacar quelas ecuaciones de ingreso de Mincer son las más utilizadas en todos los trabajos y artículos internacionales que tratan sobre la rentabilidad de la educación, 
razón por la cual serán incluidas en este trabajo, a pesar de que existen algunas críticas a este modelo; sin embargo, se ha mantenido vigente en el tiempo siendo lo bastante flexible como para incorporar algunas modificaciones en sus parámetros analíticos [www.ief.es/documentos/investigacion/seminarios/economia_ publica/2002_14Febrero.pdf]).

\section{METOdOLOGÍA}

El modelo econométrico de Mincer ${ }^{4-5}$ se fundamenta en la relación existente entre las identidades económicas y los ingresos que son obtenidos a través de la educación. En su esencia se postula una relación positiva entre el logaritmo natural (Ln) de los ingresos con los años de educación y experiencia laboral.

La clásica ecuación de ingresos de Mincer se estima por medio de los mínimos cuadrados ordinarios (MCO), siendo un modelo de tipo semilogarítmico que utiliza como variable dependiente el logaritmo natural de los ingresos y como variables independientes los años de educación formal, la experiencia laboral y el cuadrado de esta última. Los datos utilizados para su estimación provienen tradicionalmente de datos de corte transversal. ${ }^{14}$

El modelo en cuestión se puede representar de acuerdo con la siguiente ecuación de ingresos:

Donde:

$$
\text { (1) Lni }=\beta_{0}+\beta_{1} \text { ED }+\beta_{2} E P+\beta_{3} \text { Conc }+\varepsilon
$$

Lni: Logaritmo natural de los ingresos del individuo.

ED: Número de años de educación formal completa.

EP: Años de experiencia laboral.

Conc: Experiencia laboral (en años) al cuadrado (se incluye para modelar los retornos marginales decrecientes de cada año adicional de experiencia).

$\beta 1$ : Tasa de rentabilidad de la educación.

$\beta 2$ : $\quad$ Coeficiente que mide el efecto de la experiencia en los ingresos.

$\varepsilon$ : Término de perturbación aleatoria que se distribuye según una Normal $(0, \sigma \varepsilon 2)$.

La ecuación de ingresos de Mincer plantea una relación de equivalencia de los perfiles del logaritmo natural del ingreso con respecto a los distintos niveles de educación, considerando además los niveles de experiencia laboral. Cabe mencionar que la ecuación de Mincer se construye bajo los supuestos del modelo neoclásico del funcionamiento del mercado de trabajo, donde las empresas conocen la productividad marginal de cada trabajador y el proceso competitivo que hace que los salarios dependan de dicho nivel de productividad. Con tal razonamiento, el valor del coeficiente de los años de educación formal es interpretado como la tasa de rendimiento medio de un año adicional de estudio adquirido por los trabajadores. Complementariamente, el modelo considera que a medida que aumentan los años de experiencia laboral, los ingresos de los trabajadores decrecen marginalmente. Esto determina que la función de ingresos de Mincer se manifieste de una forma cóncava con relación a la experiencia de trabajo, esperándose, coherentemente, que la estimación de $\beta 2$ sea positiva y la de $\beta 3$ sea negativa.

Respecto a la variable de la ecuación de Mincer referida a la experiencia laboral, este introduce un parámetro simplificador por cuanto, en su momento, no se disponía de la experiencia efectiva de los trabajadores. Dicho parámetro permitía calcular la experiencia laboral a través de la siguiente fórmula:

$$
\text { (2) } \mathrm{X}_{\mathrm{it}}=\mathrm{Edad}_{\mathrm{it}}-\mathrm{ED}_{\mathrm{it}}-6
$$

De la cual se deduce que Mincer infería que todos los años en que un individuo no se encontraba estudiando, estaba integrado en el mercado laboral. Asimismo, suponía que la edad de ingreso al sistema educativo era a los 6 años.

El primer supuesto es cuestionable, dado que muy probablemente muchas personas que no están estudiando tampoco se encuentran en el mercado de trabajo.

El segundo supuesto es aceptable, ya que en Chile a los 6 años se ingresa al primer año de enseñanza básica.

Aunque el modelo de Mincer continúa teniendo detractores que señalan múltiples debilidades ${ }^{15}$, como el sesgo por omisión al no considerar la habilidad del trabajador en el retorno de la educación, sin embargo, en la actualidad, sus postulados siguen siendo un aporte para determinar la rentabilidad de la educación.

Este estudio pone énfasis en las diferencias en las tasas de retorno según el nivel de educación (primaria, secundaria, universidad) y de acuerdo a los grupos conformados por las edades de los individuos. Asimismo, recoge la contribución de algunos importantes autores chilenos, como Sapelli y Beyer, por sus aportes a este país, los cuales realizan ajustes a la ecuación de Mincer, considerando de esta forma las principales críticas a la fórmula propuesta por este autor, al aplicarla empíricamente, sobre las tasas de retorno de la educación para sus principales niveles: Educación Básica, Educación Media y Educación Superior en el caso de Chile.

\section{Análisis de datos}

Los datos utilizados en este estudio se obtuvieron de la encuesta de Caracterización Socioeconómica Nacional (CASEN) realizada en Chile por el Ministerio de Desarrollo Social, cuyo propósito es recabar información del estado respecto de temas como salud, vivienda, trabajo, demografía, educación e ingresos de la población. Cabe señalar que la encuesta CASEN se viene aplicando desde el año 1985 a la fecha, con una periodicidad de 2 a 3 años, siendo la última la efectuada el 2011. Esta investigación utiliza los datos recogidos por la encuesta CASEN en los años 1992, 2000, 2009 y 2011, lo que permite observar y analizar la evolución de las variables de una forma transversal y suficientemente consistente a través del tiempo.

Para poder extraer los datos requeridos por la ecuación de ingresos de Mincer desde la base de datos de la encuesta CASEN, fue necesario, en primer lugar, determinar y seleccionar el perfil de ingreso que se aplicó a la información existente, la cual debió necesariamente tener parámetros comparativos respecto de los años seleccionados por este estudio, optando por configurar la búsqueda de los ingresos en función de los quintiles de edad y tipo de educación.

En cuanto a la caracterización de los ingresos, se eligió como opción la que hace referencia a los ingresos autónomos (procedentes de sueldos, salarios y otras ganancias del trabajo independiente) por representar, junto a una mayor integralidad, la opción más utilizada en estudios previos chilenos (como los de Beyer y Sapelli).

Los análisis consideraron un rango de edad de entre 25 y 79 años como período del ciclo laboral, la media de la edad entre cada uno de los quinquenios y los niveles de estudio clasificados de la 
siguiente manera: Enseñanza Básica, Enseñanza Media y Enseñanza Superior. Para los análisis estadísticos se utilizó el software Radatam.

\section{RESULTADOS}

En la Tabla se puede observar los ingresos autónomos de los trabajadores por rango de edad y niveles de educación en el año 1992, a la vez de un factor que permite medir el valor incremental del ingreso autónomo a medida que este posee un mayor nivel de educación. Cabe destacar que se ha utilizado las siglas FB, FM y FS para los distintos factores incrementales de ingreso para la enseñanza básica, media y superior, respectivamente. Siendo la enseñanza básica el factor 1 como base.

\section{Tabla 1.}

Ingresos autónomos por quinquenios de edad y niveles de educación en Chile, 1992.

$\begin{array}{lcccccc}\text { RANGO EDAD } & \text { E. BÁSICA } & \text { FB } & \text { E. MEDIA } & \text { FM } & \text { E. SUPERIOR } & \text { FS } \\ 25-29 & 66.578,05 & 1,00 & 100.145,11 & 1,50 & 184.924,82 & 2,78 \\ 30-34 & 76.801,21 & 1,00 & 125.725,07 & 1,64 & 359.349,60 & 4,68 \\ 35-39 & 84.501,85 & 1,00 & 167.267,93 & 1,98 & 302.749,46 & 3,58 \\ 40-44 & 115.501,21 & 1,00 & 181.821,19 & 1,57 & 402.439,23 & 3,48 \\ 45-49 & 131.831,97 & 1,00 & 208.888,57 & 1,58 & 324.529,84 & 2,46 \\ 50-54 & 124.621,76 & 1,00 & 235.161,94 & 1,89 & 467.739,34 & 3,75 \\ 55-59 & 93.808,47 & 1,00 & 246.582,70 & 2,63 & 442.179,80 & 4,71 \\ 60-64 & 143.815,45 & 1,00 & 221.719,78 & 1,54 & 647.190,66 & 4,50 \\ 65-69 & 121.235,40 & 1,00 & 210.864,09 & 1,74 & 605.001,65 & 4,99 \\ 70-74 & 94.326,92 & 1,00 & 200.811,54 & 2,13 & 423.280,31 & 4,49 \\ 75-79 & 85.313,74 & 1,00 & 117.573,20 & 1,38 & 780.626,04 & 9,15\end{array}$

Fuente: Elaboración propia en base a datos de la CASEN 1992.

Los resultados denotan evidentes diferencias en cuanto a los ingresos entre los trabajadores, aspecto que depende fundamentalmente de su nivel de enseñanza, siendo la edad, al parecer, un factor no tan relevante. Por ejemplo, para el rango de edad 30-34 años, el trabajador con enseñanza media supera en más de un 60\% el ingreso de un trabajador que posee sólo enseñanza básica, pudiendo el trabajador con enseñanza superior alcanzar un ingreso autónomo cuatro veces mayor a la persona que alcanza sólo la enseñanza básica.

Gráfico 1.

Ingresos autónomos por quintiles de edad y niveles de educación en Chile, 1992.

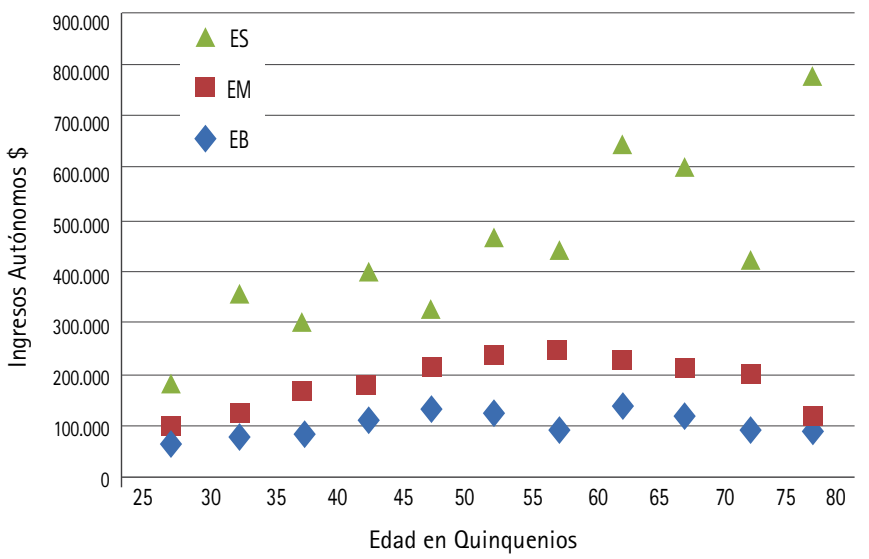

Fuente: Elaboración propia en base a datos de la CASEN 1992.
Tabla 2.

Ingresos autónomos por edad y niveles de educación en Chile, 2000.

$\begin{array}{lcccccc}\text { RANG0 EDAD } & \text { E. BÁSICA } & \text { FB } & \text { E. MEDIA } & \text { FM } & \text { E. SUPERIOR } & \text { FS } \\ 25-29 & 109.678,97 & 1,00 & 162.866,00 & 1,48 & 580.507,90 & 5,29 \\ 30-34 & 116.988,12 & 1,00 & 204.310,83 & 1,75 & 803.208,10 & 6,87 \\ 35-39 & 138.151,36 & 1,00 & 205.613,55 & 1,49 & 737.442,34 & 5,34 \\ 40-44 & 137.458,15 & 1,00 & 246.281,04 & 1,79 & 1.164 .318,04 & 8,47 \\ 45-49 & 169.621,80 & 1,00 & 291.891,93 & 1,72 & 1.220 .544,39 & 7,20 \\ 50-54 & 191.426,10 & 1,00 & 342.790,93 & 1,79 & 1.063 .219,32 & 5,55 \\ 55-59 & 184.899,12 & 1,00 & 319.770,16 & 1,73 & 1.112 .266,59 & 6,02 \\ 60-64 & 150.784,02 & 1,00 & 298.077,76 & 1,98 & 953.015,74 & 6,32 \\ 65-69 & 200.220,23 & 1,00 & 258.929,35 & 1,29 & 1.239 .401,15 & 6,19 \\ 70-74 & 125.836,08 & 1,00 & 530.760,22 & 4,22 & 910.042,64 & 7,23 \\ 75-79 & 169.109,05 & 1,00 & - & - & 637.608,94 & 3,77\end{array}$

Fuente: Elaboración propia en base a datos de la CASEN 2000.

En el Gráfico 1 se puede observar cómo los ingresos autónomos de trabajadores con enseñanza superior crecen a lo largo de casi toda la vida laboral, en comparación a los trabajadores con enseñanza básica y media, donde sus ingresos comienzan a disminuir una vez que han llegado a la mitad de sus ciclos laborales.

La Tabla 2 muestra los resultados correspondientes al año 2000, donde se pueden apreciar, al igual que para el año 1992, mayores diferencias de ingresos entre los trabajadores con distintos niveles de enseñanza. Por ejemplo, para el rango de edad 30-34 años se puede observar que el trabajador con enseñanza media supera sus ingresos autónomos en un 75\% respecto del trabajador con sólo enseñanza básica, incrementándose esta diferencia en más de 5 veces cuando el trabajador posee educación superior. Llegando, incluso, en el rango 40-44, a superar en más de 8 veces el ingreso en comparación al trabajador con sólo enseñanza básica.

El Gráfico 2 muestra la dispersión de los datos respecto del ingreso autónomo en el año 2000, donde se pueden ver las diferencias en los niveles de ingreso entre la educación superior y los demás niveles de enseñanza. Observándose, a su vez, que los ingresos de los trabajadores con educación superior comienzan a tener una disminución en sus niveles, cuando estos se aproximan a la mitad del ciclo laboral, aspecto también observado en los trabajadores con enseñanza básica y media. Por otra parte, en el 2009, los análisis desprenden que las diferencias de ingresos persisten y siguen siendo muy favorables para trabajadores con enseñanza superior, lo cual es coherente con lo planteado por Mizala y Romaguera. ${ }^{16}$ No obstante, estas diferencias son signi-

Gráfico 2.

Dispersión de ingresos por edad y niveles de educación en Chile, 2000.

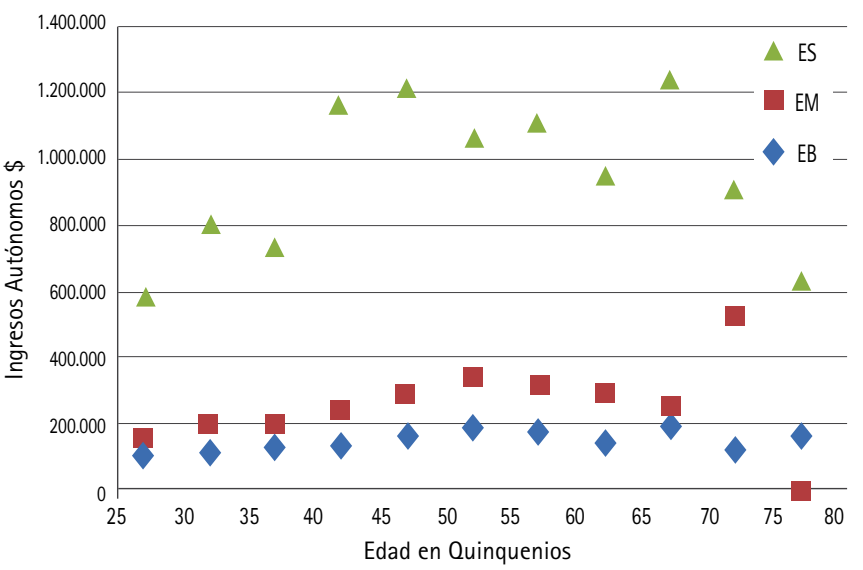

Fuente: Elaboración propia en base a datos de la CASEN 2000. 
ficativamente menores a las observadas en el año 2000. La Tabla 3 da cuenta de aquello, pudiéndose apreciar, a su vez, una excepción en el rango de edad entre 60 y 64 años. Diferencias particulares, claramente señaladas en el Gráfico 3, en el cual también se puede observar, respecto del comportamiento de los ingresos, un patrón muy similar a lo sucedido en el año 2000 relativo al aumento de la edad de los trabajadores, donde los ingresos comienzan a decrecer una vez alcanzada la medianía del ciclo laboral.

Tabla 3.

Ingresos autónomos, edad y niveles de educación en Chile, 2009.

$\begin{array}{lcccccc}\text { RANGO EDAD } & \text { E. BÁSICA } & \text { FB } & \text { E. MEDIA } & \text { FM } & \text { E. SUPERIOR } & \text { FS } \\ 25-29 & 199.537,89 & 1,00 & 263.968,69 & 1,32 & 784.901,53 & 3,93 \\ 30-34 & 233.718,38 & 1,00 & 294.018,92 & 1,26 & 852.309,91 & 3,65 \\ 35-39 & 253.707,17 & 1,00 & 330.047,32 & 1,30 & 1.081 .320,90 & 4,26 \\ 40-44 & 242.201,51 & 1,00 & 366.530,81 & 1,51 & 1.154 .893,31 & 4,77 \\ 45-49 & 240.617,76 & 1,00 & 375.765,64 & 1,56 & 1.267 .745,78 & 5,27 \\ 50-54 & 265.524,01 & 1,00 & 390.039,20 & 1,47 & 1.660 .341,94 & 6,25 \\ 55-59 & 256.139,57 & 1,00 & 400.937,01 & 1,57 & 1.137 .002,42 & 4,44 \\ 60-64 & 244.774,43 & 1,00 & 387.192,70 & 1,58 & 2.362 .751,94 & 9,65 \\ 65-69 & 204.406,50 & 1,00 & 357.874,49 & 1,75 & 1.107 .074,44 & 5,42 \\ 70-74 & 171.547,67 & 1,00 & 307.446,26 & 1,79 & 796.221,48 & 4,64 \\ 75-79 & 147.272,59 & 1,00 & 297.574,26 & 2,02 & 468.689,84 & 3,18\end{array}$

Fuente: Elaboración propia en base a datos de la CASEN 2009.

Finalmente se presentan los resultados de los análisis de datos con la información más recientes de la encuesta CASEN2011 ${ }^{17}$ (Tabla 4 y Gráfico 3), donde se puede inferir un comportamiento de los ingresos muy similar a lo detectado en el año 2009, con la salvedad de que los ingresos de los trabajadores con educación superior crecen de forma mucho más acelerada en los primeros quinquenios del ciclo laboral de lo observado en años anteriores, decreciendo fuertemente estos una vez superado los 55 años de edad.

Tabla 4.

Ingresos autónomos, edad y niveles de educación en Chile, 2011.

\begin{tabular}{|lcccccc}
\hline RANGO EDAD & E. BÁSICA & FB & E. MEDIA & FM & E. SUPERIOR & FS \\
\hline 27 & $228.900,89$ & 1,00 & $303.611,24$ & 1,33 & $728.510,21$ & 3,18 \\
32 & $226.982,27$ & 1,00 & $341.054,59$ & 1,50 & $948.926,51$ & 4,18 \\
37 & $252.435,78$ & 1,00 & $332.083,87$ & 1,32 & $1.027 .308,12$ & 4,07 \\
42 & $259.752,96$ & 1,00 & $361.582,11$ & 1,39 & $1.244 .759,82$ & 4,79 \\
47 & $312.778,31$ & 1,00 & $434.640,44$ & 1,39 & $1.263 .112,48$ & 4,04 \\
52 & $271.170,05$ & 1,00 & $453.310,43$ & 1,67 & $1.266 .817,71$ & 4,67 \\
57 & $282.383,57$ & 1,00 & $492.562,66$ & 1,74 & $1.507 .890,18$ & 5,34 \\
62 & $273.293,71$ & 1,00 & $473.349,51$ & 1,73 & $1.109 .489,22$ & 4,06 \\
67 & $232.146,24$ & 1,00 & $395.163,97$ & 1,70 & $788.162,69$ & 3,40 \\
72 & $196.919,14$ & 1,00 & $422.495,64$ & 2,15 & $783.703,98$ & 3,98 \\
77 & $190.553,76$ & 1,00 & $308.737,15$ & 1,62 & $741.622,43$ & 3,89
\end{tabular}

Fuente: Elaboración propia en base a datos de la CASEN 2011.

Cabe destacar que los antecedentes empíricos recabados para el caso de Chile permiten afirmar que se cumplen los supuestos del modelo de ingresos de Mincer, ya que a partir de los datos se infiere que la función de ingresos es cóncava en relación a la experiencia de los trabajadores.

Retomando la ecuación (1) expuesta en este trabajo:

$$
\text { Lni }=\beta_{0}+\beta_{1} \mathrm{ED}+\beta_{2} \mathrm{EP}+\beta_{3} \text { Conc }+\varepsilon
$$

Se realizaron los cálculos logarítmicos de los ingresos autónomos, que en principio no discriminan entre los niveles educacionales de
Gráfico 3.

Ingresos autónomos, edad y niveles de educación en Chile, 2011.

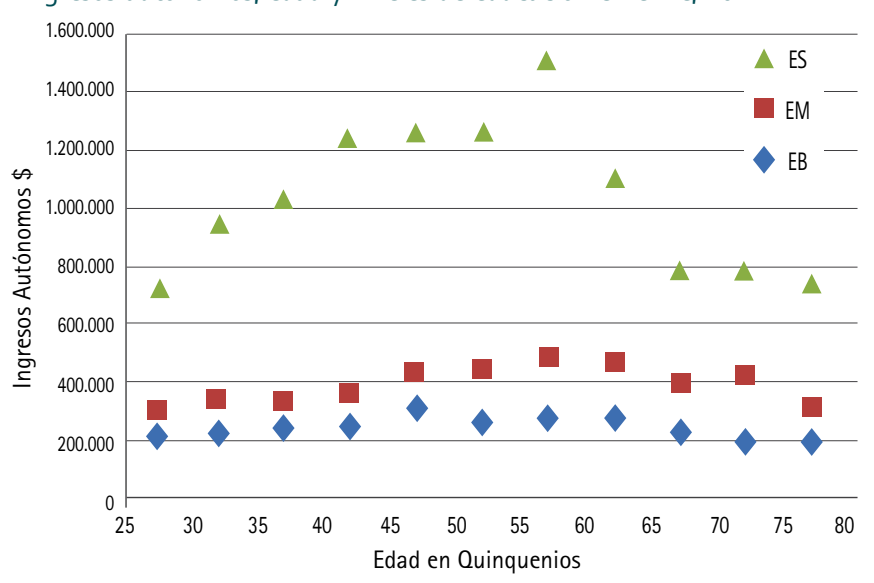

Fuente: Elaboración propia en base a datos de la CASEN 2011.

los trabajadores, obteniéndose por ende una medida general de la rentabilidad de la educación en Chile para los distintos años en análisis. Posteriormente, y tras la utilización del software de análisis econométrico Eviews 7.0, se calculó la regresión respectiva para el año 1992. En la Tabla 5 se puede apreciar un resumen de los resultados de la regresión múltiple efectuada, en donde se puede observar que la serie ED (que representa los años de educación formal) es significativa con 19,26939 en su tau, con una probabilidad asociada menor a 0,05 (t-calculado). Cabe destacar que, en este caso, como la tau es menor a 0,05 con una probabilidad de 0,000 , se rechaza la hipótesis nula, pudiéndose afirmar que el coeficiente asociado a la variable en estudio es significativo, lo cual, tomando como referencia el modelo de Mincer, pudiese determinar que la rentabilidad de la educación formal en Chile para el año 1992 fue de un 16,75\%, donde la variable experiencia (EP) explicaría en un 6,5\% los ingresos autónomos de los trabajadores en ese año. Adicionalmente, se puede observar que el coeficiente de determinación es muy alto (en torno a un 0,933529), lo que pudiese indicar que las variables Educación + Experiencia, explicarían en un 93\% la variable Ingresos Autónomos.

La Tabla 6 muestra la rentabilidad general de la educación formal en el año 2000, donde se puede apreciar que esta aumenta a un $21 \%$ y la participación de la experiencia en la generación de los ingresos autónomos disminuye a un 3,7\%. Asimismo, al considerar los datos del año 2009 (Tabla 7), se puede observar una disminución

Tabla 5.

Resumen de la regresión general de Mincer, Chile, 1992.

$\begin{array}{lcccc}\text { Variable } & \text { Coefficient } & \text { Std. Error } & \text { t-Statistic } & \text { Prob. } \\ \text { C } & 9,162061 & 0,169616 & 54,01647 & 0,0000 \\ \text { ED } & 0,167545 & 0,008695 & 19,26939 & 0,0000 \\ \text { EP } & 0,065249 & 0,008488 & 7,687010 & 0,0000 \\ \text { CONC } & -0,000891 & 0,000132 & -6,772999 & 0,0000 \\ \text { R-squared } & 0,933529 & \text { Mean dependentvar } & 12,18203 \\ \text { Adjusted R-squared } & 0,926653 & \text { S. D. dependentvar } & 0,672926 \\ \text { S.E. of regression } & 0,182247 & \text { Akaikeinfocriterion } & -0,453698 \\ \text { Sum squaredresid } & 0,963202 & \text { Schwarzcriterion } & -0272303 \\ \text { Log likelihood } & 11,48602 & \text { Hannan-Quinncriter, } & -0,392664 \\ \text { F-statistic } & 135,7598 & \text { Durbin-Watson stat } & 2,400670 \\ \text { Prob(F-statistic) } & 0,000000 & & \end{array}$

Fuente: Reporte regresión software Eviews 7. 
de la rentabilidad general de la educación, cayendo ésta a un 15,8\%, porcentaje inclusive inferior a lo obtenido en el año 1992 $(16,7 \%)$. Por su parte, la experiencia se mantiene, con una participación en la generación de ingresos autónomos en torno al 4\%, similar al año 2000. Cabe destacar que para todas las series de variables (Educación y Experiencia), se observan valores de significancia en su tau, con una probabilidad asociada al t-calculado menor a 0,05. Finalmente, tanto para el año 2000 como para el año 2009, se aprecia un coeficiente de determinación muy alto, que explicaría la variable Ingresos Autónomos en un 93\% (2000) y en un 87,5\% (2009) respectivamente (Tablas 6 y 7).

Tabla 6.

Resumen de la regresión general de Mincer, Chile, 2000.

$\begin{array}{lcccc}\text { Variable } & \text { Coefficient } & \text { Std. Error } & \text { t-Statistic } & \text { Prob. } \\ \text { C } & 9,562235 & 0,222623 & 42,95254 & 0,0000 \\ \text { ED } & 0,209764 & 0,011203 & 18,72355 & 0,0000 \\ \text { EP } & 0,037347 & 0,012264 & 3,045245 & 0,0053 \\ \text { CONC } & -0,000499 & 0,000207 & -2,412659 & 0,0232 \\ \text { R-squared } & 0,931364 & \text { Mean dependentvar } & 12,73193 \\ \text { Adjusted R-squared } & 0,923444 & \text { S.D. dependentvar } & 0,826721 \\ \text { S.E. of regression } & 0,228743 & \text { Akaikeinfocriterion } & 0,011130 \\ \text { Sum squaredresid } & 1,360407 & \text { Schwarzcriterion } & 0,197957 \\ \text { Log likelihood } & 3,833047 & \text { Hannan-Quinncriter, } & 0,070898 \\ \text { F-statistic } & 117,6029 & \text { Durbin-Watson stat } & 2,597149 \\ \text { Prob(F-statistic) } & 0,000000 & & \end{array}$

Fuente: Reporte regresión software Eviews 7.

Tabla 7.

Resumen de la regresión general de Mincer, Chile, 2009.

$\begin{array}{lcccc}\text { Variable } & \text { Coefficient } & \text { Std. Error } & \text { t-Statistic } & \text { Prob. } \\ \text { C } & 10,61826 & 0,236616 & 44,87550 & 0,0000 \\ \text { ED } & 0,158469 & 0,011821 & 13,40595 & 0,0000 \\ \text { EP } & 0,040353 & 0,012479 & 3,233714 & 0,0030 \\ \text { CONC } & -0,000712 & 0,000194 & -3,677773 & 0,0010 \\ \text { R-squared } & 0,875672 & \text { Mean dependentvar } & 12,97188 \\ \text { Adjusted R-squared } & 0,862810 & & \text { S.D. dependentvar } & 0,725764 \\ \text { S.E. of regression } & 0,268817 & \text { Akaikeinfocriterion } & 0,323638 \\ \text { Sum squaredresid } & 2,095610 & \text { Schwarzcriterion } & 0,505033 \\ \text { Log likelihood } & -1,340032 & \text { Hannan-Quinncriter, } & 0,384672 \\ \text { F-statistic } & 68,08462 & \text { Durbin-Watson stat } & 2,285147 \\ \text { Prob(F-statistic) } & 0,000000 & & \end{array}$

Fuente: Reporte regresión software Eviews 7.

Cabe destacar que en otras investigaciones acerca de la rentabilidad de la educación en Chile, como las de Sapelli, si bien ocupan similares metodologías a la utilizada en este estudio, presentan algunas diferencias en cuanto a la información analizada. Por ejemplo, los estudios de Sapelli utilizan años de escolarización para determinar los niveles de enseñanza, lo que implica incorporar datos de ingresos de trabajadores con niveles de enseñanza completa e incompleta, asumiendo, en consecuencia, que para alcanzar la educación básica completa se deben tener 8 años de escolarización, para enseñanza media completa 12 años de escolarización y para enseñanza superior completa contar con al menos 17 años de escolarización. Consideraciones distintas a las estipuladas en el presente estudio, ya que se utilizaron sólo los datos de trabajadores con educación completa para los distintos niveles de enseñanza (Básica, Media y Superior), lo cual permitió analizar la rentabilidad de la educación de cada nivel educativo efectivamente completado, en coherencia con
Tabla 8.

Resultados de los parámetros de la ecuación de Mincer, respecto de Sapelli y del presente estudio, 1990-2009.

$\begin{array}{lccccc}\text { Resultados } & \text { Sapelli } & \text { Sapelli } & \text { Estudio } & \text { Estudio } & \text { Estudio } \\ & 1990 & 1998 & 1992 & 2000 & 2009 \\ \text { Educación } & 11,4 \% & 13,2 \% & 16 \% & 21 \% & 15,8 \% \\ \text { Experiencia } & 3,0 \% & 3,2 \% & 6,5 \% & 3,7 \% & 4,0 \%\end{array}$

Fuente: Elaboración propia en base a antecedentes de la investigación.

los objetivos formulados. No obstante, los resultados de Sapelli no se alejan de los obtenidos en este estudio.

En la Tabla 8 se comparan los parámetros de la ecuación de Mincer entre los trabajos de Sapelli y los hallados en esta investigación, donde se puede observar que los porcentajes de rentabilidad obtenidos en este estudio son algo superiores a los presentados por Sapelli; resultado probable, ya que al incluir los distintos niveles de enseñanza se aporta mayores retornos de ingresos al mercado laboral, en función de quien tiene un nivel de enseñanza incompleto. ${ }^{18}$ Cabe destacar que para la variable experiencia, las tasas son similares a excepción del año 1992. Asimismo, se puede observar que en este estudio el punto más alto de rentabilidad de la educación se alcanza en el año 2000, decreciendo posteriormente a lo largo del tiempo, manteniéndose en torno al 15\%. Cabe señalar que existen antecedentes empíricos en Chile de estudios de ingresos desde el año 1960, por ejemplo, el estudio de Riveros ${ }^{19}$, cuyos porcentajes de rentabilidad de la educación y de la experiencia se exponen en la Tabla 9.

\section{Tabla 9.}

Resultados de los parámetros de la ecuación de Mincer, años1965-1978.

$\begin{array}{lccc}\text { Resultados } & 1965 & 1972 & 1978 \\ \text { Educación } & 12,6 \% & 12,8 & 15,7 \% \\ \text { Experiencia } & 5,0 \% & 5,2 & 6,4 \%\end{array}$

Fuente: Elaboración propia en base a antecedentes de la investigación de L. Riveros.

En ella se puede observar un cierto crecimiento tanto de la rentabilidad de la educación como en la experiencia, obteniendo esta última en promedio porcentajes más altos de los observados por Sapelli y los arrojados por este estudio, lo cual parece ser coherente con la evolución que ha experimentado el mercado laboral de Chile, donde cada vez se valora menos la experiencia respecto de los estudios formales realizados a la hora de determinarse los salarios.

\section{Cálculo de la rentabilidad de la educación según el nivel educativo}

Si bien es cierto que la rentabilidad general de la educación en Chile es un indicador muy importante a la hora de evaluar los resultados económicos del sistema educativo, sobre todo si se considera la creciente inversión pública que se ha venido realizando en las últimas décadas, no es menos cierto que sería aún más útil para la evaluación de las políticas públicas en educación que la rentabilidad se desagregara por nivel educativo o enseñanza, que en Chile se configuran básicamente en tres niveles: básica, media y superior, donde la enseñanza básica corresponde a 8 años de escolaridad, la media a 4 años y la superior entre 3 años (carreras técnicas) y 7 años (carreras profesionales) de enseñanza. Cabe señalar que para efectos de este trabajo se consideró un período regular de 5 años como tiempo de duración del nivel superior, tiempo promedio de duración de las carreras universitarias en Chile, a la vez de no contemplar en los análisis la enseñanza pre-básica o enseñanza parvularia, por el reducido tiempo de desarrollo que este nuevo nivel ha tenido en Chile. 
Para poder desagregar los niveles educativos e incorporarlos a la ecuación de ingreso de Mincer, se utilizó el método spline ${ }^{20}$, el cual permitió incorporar las variables adicionales a la ecuación para explicar los ingresos dependiendo del nivel de enseñanza. Por ejemplo, en el caso de un trabajador con enseñanza media, la información ingresada en la ecuación de Mincer consideró 8 años de educación básica (EB), 4 años de educación media (EM) y 0 años de educación superior (ES).

En función de lo anterior, la ecuación de Mincer quedó formulada de la siguiente manera:

$$
\text { Lni }=\beta_{0}+\beta_{1} E B+\beta_{2} E M+\beta_{3} E S+\beta_{4} E P+\beta_{5} \text { Conc }+\varepsilon
$$

Donde:

Lni: Logaritmo natural de los ingresos del individuo.

EB: Número de años de educación básica formal completada.

EM: Número de años de educación media formal completada.

ES: Número de años de educación superior formal completada.

EP: Años de experiencia laboral.

Conc: Experiencia laboral (en años) al cuadrado, se incluye para modelar los retornos marginales decrecientes de cada año adicional de experiencia.

$\varepsilon$ : $\quad$ Perturbación aleatoria que se distribuye según una normal

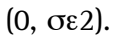

$\beta 1, \beta 2$ y $\beta 3$ : Tasas de rentabilidad para cada nivel educativo.

$\beta 4$ : Coeficiente que mide el efecto de la experiencia en los ingresos.

35 : Coeficiente que mide el retorno marginal decreciente de la experiencia.

A continuación se muestran los resultados obtenidos en la nueva formulación de la ecuación de ingresos de Mincer, desagregada por niveles de educación para los años 1992, 2000 y 2009, utilizando los mismos datos de corte transversal contemplados en la ecuación original. En la Tabla 10 se puede observar, para el año 1992, que las rentabilidades asociadas a los distintos niveles educacionales fueron de un 8\% para educación básica, 14\% para educación media y un 19\% para la educación superior. Teniendo el factor experiencia un 6\% de incidencia en el nivel de ingresos de los trabajadores. Se destaca también el coeficiente de determinación que supera el 90\%, lo cual indicaría que las variables desagregadas en la ecuación explicarian en gran medida el comportamiento de los ingresos de los trabajadores.

Las rentabilidades obtenidas dan cuenta de un mayor ingreso asignado por el mercado laboral a los trabajadores con mayores niveles educativos. En particular, la diferencia entre enseñanza media y

Tabla 10.

Resumen de datos de la nueva regresión de la ecuación de Mincer, Chile 1992.

$\begin{array}{lcccc}\text { Variable } & \text { Coefficient } & \text { Std. Error } & \text { t-Statistic } & \text { Prob. } \\ \text { C } & 9,900013 & 1,261394 & 7,848471 & 0,0000 \\ \text { EB } & \mathbf{0 , 0 8 0 9 0 5} & 0,152933 & 0,529021 & 0,6011 \\ \text { EM } & \mathbf{0 , 1 4 1 7 3 2} & 0,019217 & 7,375517 & 0,0000 \\ \text { ES } & \mathbf{0 , 1 8 8 4 0 5} & 0,015614 & 12,06666 & 0,0000 \\ \text { EP } & \mathbf{0 , 0 6 3 3 0 0} & 0,009377 & 6,750518 & 0,0000 \\ \text { CONC } & -0,000844 & 0,000158 & -5,353779 & 0,0000 \\ \text { R-squared } & \mathbf{0 , 9 3 9 5 2 6} & \text { Mean dependentvar } & 12,18203 \\ \text { Adjusted R-squared } & 0,928327 & \text { S.D. dependentvar } & 0,672926 \\ \text { S.E. of regression } & 0,180154 & \text { Akaikeinfocriterion } & -0,427043 \\ \text { Sum squaredresid } & 0,876299 & \text { Schwarzcriterion } & -0,154950 \\ \text { Log likelihood } & 13,04620 & \text { Hannan-Quinncriter. } & -0,335492 \\ \text { F-statistic } & 83,89471 & \text { Durbin-Watson stat } & 2,386671 \\ \text { Prob(F-statistic) } & 0,000000 & & \end{array}$

Fuente: Reporte regresión software Eviews 7. enseñanza básica es de seis puntos porcentuales de rentabilidad; en cambio, la diferencia entre enseñanza superior y enseñanza media sólo es de cinco puntos porcentuales. En términos relativos, la asignación de un mayor ingreso, por poseer una educación media, es de un 75\%, respecto de la educación básica. En cambio, la asignación de un mayor ingreso por poseer una educación superior, es de un $36 \%$ respecto de la educación media.

Cabe señalar que los estudios de Sapelli arrojaron resultados muy similares a los del presente estudio respecto del año 1990, apreciándose sólo una mayor diferencia para el nivel educativo enseñanza básica. Por otra parte, los estudios de Beyer respecto del año 1994 se distancian un poco más, obteniendo algunas rentabilidades menores, sobre todo para el nivel educativo de enseñanza media. En la Tabla 11 se presentan los resultados de los estudios de forma comparativa.

Tabla 11.

Rentabilidad por nivel de enseñanza, respecto de Sapelli y del presente estudio, 1990-2094.

$\begin{array}{lccc}\text { Nivel de Enseñanza } & \text { Sapelli } 1990 & \text { Estudio } 1992 & \text { Beyer } 1994 \\ \text { E. Básica } & 6 \% & 8 \% & 6 \% \\ \text { E. Media } & 13 \% & 14 \% & 4 \% \\ \text { E. Superior } & 19 \% & 19 \% & 12 \%\end{array}$

Fuente: Elaboración propia en base a antecedentes de la investigación.

Cabe destacar que los estudios de Beyer se enfocaron en la desigualdad de los ingresos en Chile, utilizando la ecuación de Mincer para demostrar empíricamente que la mayor desigualdad se encontraba en la clase socioeconómica media, ya que la educación superior era un nivel de enseñanza casi exclusiva del sector socioeconómico alto. Además, se contempló un universo compuesto sólo por hombres asalariados de entre 25 y 59 años, e incorporando a la ecuación una variable adicional a los niveles educacionales, denominada Jefes de Hogar. A pesar de estas diferencias metodológicas -y otras menores no mencionadas-, es interesante apreciar el mayor ingreso que las personas con educación superior recibe, por sobre los demás niveles educativos.

Asimismo, y respecto del año 2000, en la Tabla 12 se muestran los resultados obtenidos en la presente investigación, donde se puede apreciar cómo la rentabilidad para el nivel de enseñanza básica se mantiene en un 6\%, para enseñanza media crece a un 14\% y para la enseñanza superior alcanza un 27\%, esto es, 8 puntos porcentuales más que la rentabilidad obtenida para el año 1992.

Esta evolución significativa de la rentabilidad de la educación superior se evidencia para un período en que el crecimiento econó-

Tabla 12.

Resumen de la nueva regresión de la Ecuación de Mincer, Chile, 2000.

$\begin{array}{lcccc}\text { Variable } & \text { Coefficient } & \text { Std. Error } & \text { t-Statistic } & \text { Prob. } \\ \text { C } & 10,77991 & 1,076329 & 10,01544 & 0,0000 \\ \text { EB } & \mathbf{0 , 0 6 3 7 1 5} & 0,135279 & 0,470992 & 0,6419 \\ \text { EM } & \mathbf{0 , 1 4 8 7 4 4} & 0,022011 & 6,757842 & 0,0000 \\ \text { ES } & \mathbf{0 , 2 6 5 7 3 5} & 0,016967 & 15,66205 & 0,0000 \\ \text { EP } & \mathbf{0 , 0 3 6 7 0 9} & 0,010151 & 3,616346 & 0,0014 \\ \text { CONC } & -0,000443 & 0,000180 & -2,465891 & 0,0212 \\ \text { R-squared } & 0,958229 & \text { Mean dependentvar } & 12,73193 \\ \text { Adjusted R-squared } & 0,949526 & \text { S.D. dependentvar } & 0,826721 \\ \text { S.E. of regression } & 0,185734 & \text { Akaikeinfocriterion } & -0,352147 \\ \text { Sum squaredresid } & 0,827930 & \text { Schwarzcriterion } & -0,071908 \\ \text { Log likelihood } & 11,28221 & \text { Hannan-Quinncriter. } & -0,262496 \\ \text { F-statistic } & 110,1115 & \text { Durbin-Watson stat } & 2,564922 \\ \text { Prob(F-statistic) } & 0,000000 & & \end{array}$

Fuente: Reporte regresión software Eviews 7. 
Tabla 13.

Rentabilidad por nivel de enseñanza, respecto de Sapelli y del presente estudio, 1998-2000.

$\begin{array}{lcc}\text { Nivel de Enseñanza } & \text { Sapelli } 1998 & \text { Estudio } 2000 \\ \text { E. Básica } & 7 \% & 6 \% \\ \text { E. Media } & 14 \% & 15 \% \\ \text { E. Superior } & 23 \% & 27 \%\end{array}$

Fuente: Elaboración propia en base a antecedentes de la investigación.

Tabla 14.

Resumen de la nueva regresión de la ecuación de Mincer, Chile, 2009.

$\begin{array}{lcccc}\text { Variable } & \text { Coefficient } & \text { Std. Error } & \text { t-Statistic } & \text { Prob. } \\ \text { C } & 11,66719 & 0,587417 & 19,86185 & 0,0000 \\ \text { EB } & \mathbf{0 , 0 2 2 2 1 9} & 0,078974 & 0,281347 & 0,7806 \\ \text { EM } & \mathbf{0 , 1 0 2 9 7 6} & 0,026536 & 3,880566 & 0,0006 \\ \text { ES } & \mathbf{0 , 2 2 7 5 6 4} & 0,018007 & 12,63724 & 0,0000 \\ \text { EP } & \mathbf{0 , 0 4 4 8 6 2} & 0,009835 & 4,561463 & 0,0001 \\ \text { CONC } & -0,000754 & 0,000151 & -4,987323 & 0,0000 \\ \text { R-squared } & 0,930454 & \text { Mean dependentvar } & 12,97179 \\ \text { Adjusted R-squared } & \mathbf{0 , 9 1 7 5 7 5} & \text { S.D. dependentvar } & 0,725733 \\ \text { S.E. of regression } & 0,208356 & \text { Akaikeinfocriterion } & -0,136170 \\ \text { Sum squaredresid } & 1,172132 & \text { Schwarzcriterion } & 0,135923 \\ \text { Log likelihood } & 8,246797 & \text { Hannan-Quinncriter. } & -0,044619 \\ \text { F-statistic } & 72,24635 & \text { Durbin-Watson stat } & 1,954598 \\ \text { Prob(F-statistic) } & 0,000000 & & & \end{array}$

Fuente: Reporte regresión software Eviews 7.

mico de Chile fue acompañado con un mayor acceso a la educación superior, posibilitando a la clase media tener mayor participación en los niveles de ingreso.

Los resultados de Sapelli respecto del año 1998 fueron muy similares a los obtenidos en la presente investigación, respecto el año 2000, en especial, para el nivel educativo de enseñanza básica y media. Sin embargo, para la enseñanza superior los resultados contemplan diferencias, siendo la rentabilidad obtenida por Sapelli de un 23\% y la de este estudio de un 27\%. En la Tabla 13 se da cuenta de estos resultados.

Cabe destacar que las rentabilidades asociadas a la educación superior en Chile siguen siendo muy importantes, lo cual da cuenta de una evolución creciente desde el año 1990 a la fecha. Finalmente, y respecto del año 2009, las rentabilidades obtenidas en este estudio, para los distintos niveles de enseñanza, decrecen respecto del año 2000, fluctuando la pérdida de rentabilidad para todos los niveles educativos entre un 4 y un 5 por ciento (Tabla 14).

Lo expuesto podría ser una clara señal de que el crecimiento de la rentabilidad de la educación para sus distintos niveles de enseñanza observado en la década de los noventa comienza a decrecer durante la primera década del 2000, pudiendo incluso disminuir aún más en los próximos años. Para poder tener una mejor constatación de lo planteado, se utilizó la información más reciente de la encuesta CASEN $2011^{17}$ para determinar las rentabilidades de los niveles educativos a través de la ecuación de ingresos de Mincer.

En la Tabla 15, se puede observar que la rentabilidad de la enseñanza básica y media aumentan en un punto porcentual, respecto del año 2009. Por el contrario, la rentabilidad de la educación superior disminuye casi 4 puntos porcentuales respecto de igual año.

Aspecto a considerar al momento de evaluar el costo de oportunidad de los recursos que actualmente se están incorporando a la educación superior en Chile. Como una forma de visualizar la evolución de las rentabilidades de los niveles de enseñanza en Chile, se muestra en la Tabla 16 y en el Gráfico 4 los períodos de crecimiento y decrecimiento de las respectivas rentabilidades en Chile entre los años 1992 y 2011.
Tabla 15.

Resumen de la nueva regresión de la ecuación de Mincer, Chile, 2011.

$\begin{array}{lcccc}\text { Variable } & \text { Coefficient } & \text { Std. Error } & \text { t-Statistic } & \text { Prob. } \\ \text { C } & 11,71787 & 0,724122 & 16,18218 & 0,0000 \\ \text { EB } & \mathbf{0 , 0 3 6 7 2 2} & 0,085771 & 0,428141 & 0,6719 \\ \text { EM } & \mathbf{0 , 1 1 4 9 2 5} & 0,015740 & 7,301341 & 0,0000 \\ \text { ES } & \mathbf{0 , 1 9 1 9 4 1} & 0,012391 & 15,49076 & 0,0000 \\ \text { EP } & \mathbf{0 , 0 3 5 6 8 7} & 0,007061 & 5,053992 & 0,0000 \\ \text { CONC } & -0,000584 & 0,000113 & -5,173160 & 0,0000 \\ \text { R-squared } & 0,955934 & \text { Mean dependentvar } & 13,03349 \\ \text { Adjusted R-squared } & 0,947774 & \text { S.D. dependentvar } & 0,627587 \\ \text { S.E. of regression } & 0,143423 & \text { Akaikeinfocriterion } & -0,883076 \\ \text { Sum squaredresid } & 0,555392 & \text { Schwarzcriterion } & -0,610983 \\ \text { Log likelihood } & 20,57075 & & \text { Hannan-Quinncriter. } & -0,791525 \\ \text { F-statistic } & 117,1442 & \text { Durbin-Watson stat } & 2,137428 \\ \text { Prob(F-statistic) } & 0,000000 & & \end{array}$

Fuente: Reporte regresión software Eviews 7.

Tabla 16.

Rentabilidad por nivel de enseñanza, Chile, 1992-2011.

$\begin{array}{lcccc}\text { Nivel de Enseñanza } & \text { Año 1992 } & \text { Año 2000 } & \text { Año 2009 } & \text { Año 2011 } \\ \text { E. Básica } & 8 \% & 6 \% & 2 \% & 4 \% \\ \text { E. Media } & 14 \% & 15 \% & 10 \% & 11 \% \\ \text { E. Superior } & 19 \% & 27 \% & 23 \% & 19 \%\end{array}$

Fuente: Elaboración propia en base a antecedentes de la investigación.

Gráfico 4.

Evolución de la rentabilidad por nivel de enseñanza, Chile, 1992-2011.

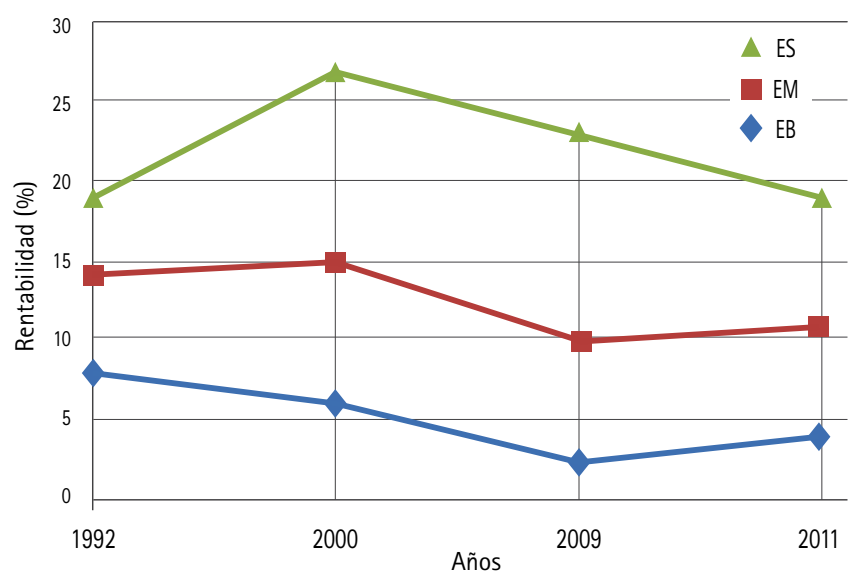

Fuente: Elaboración propia en base a resultados de la investigación.

El Gráfico 4 muestra que entre los años 2000 y 2009 las rentabilidades de todos los niveles educativos decrecen, siendo sólo los niveles de enseñanza básica y media los que vuelven a aumentan a partir del año 2009. En cambio, la rentabilidad de la educación superior sigue decreciendo, alcanzado su peak en el año 2000. Asimismo, se puede observar que entre 1992 y el 2000 hay un comportamiento más irregular entre los niveles educativos, destacándose el gran crecimiento de la rentabilidad de la educación superior, que puede ser aún mayor dependiendo de la carrera universitaria y la institución educativa. ${ }^{21}$

\section{DISCUSIÓN}

Respecto a la rentabilidad del trabajo, esta investigación pudo constatar diferencias significativas en los ingresos de los trabajadores dependiendo fundamentalmente del nivel de enseñanza. En particular, la enseñanza superior es la más rentable para todos los 
años estudiados, alcanzando su peak en el año 2000 con un 27\%. A partir del cual la rentabilidad comienza lentamente a decrecer, pero sin dejar de ser la más rentable, disminuyendo a un $23 \%$ el 2009 y a un 19\% el 2011.

La edad contemplada como experiencia favorece los ingresos en forma creciente hasta la mitad del ciclo de vida laboral (entre los 50 y 55 años), a partir del cual la experiencia como causante de los ingresos comienza a ser cada vez menos gravitante.

$\mathrm{Al}$ parecer uno de los factores que contribuye a la gran desigualdad económica y social en Chile es, precisamente, la gran diferencia entre los ingresos de los trabajadores, que para el período bajo estudio no parece haber mejorado sustancialmente. Prueba de ello es que la razón 10/10 que relaciona el 10\% de la población con mayores ingresos, con el 10\% de la población con menores ingresos, ha ido creciendo, sobrepasando en 34 veces el año 2000 y en 46 veces el año 2009. Siendo que el año 1990 dicho indicador sólo alcanzaba 30 veces. ${ }^{11}$

La desigualdad social y económica en Chile, no sólo comporta el nivel educacional de los trabajadores, sino también la procedencia social e institucional. Esto contempla el lugar en que habita, su círculo familiar y social y la institución educativa que lo formó. De esta forma, se hace muy necesario avanzar en Chile hacia el análisis de la rentabilidad social, contemplando el capital humano inicial y de procedencia socioeconómica de la población bajo estudio. ${ }^{22}$

\section{REFERENCIAS}

1. Becker G. El Capital Humano Madrid: Alianza; 1983.

2. Schultz TW. Investment in Human Capital. Am Econ Rev.1961;(51):1-17.

3. Brunner J, Elacqua G. Informe Capital Humano en Chile. Santiago de Chile: Universidad Adolfo lbáñez; 2003.

4. Mincer J. Investment in Human Capital and Personal Income Distribution.J Polit Econ.1958; 66(4):281-302.

5. Mincer J.Schooling, Experience and Earnings. New York: National Bureau of Economic Research;1974.

6. Ben-Porath Y. The Production of Human Capital and the Life Cycle of Earnings. J Polit Econ. 1967;75(4):352-365.

7. Willis R. Wage determinants: a survey and reinterpretation of human capital earnings functions. In: Ashenfelter O, Layard R,editores. Handbook of Labor Economics; Vol. I. Amsterdam: Elsevier; 1987. p.525-602.

8. Heckman J, Lochner L, Todd P. Fifty years of Mincer Earnings regressions. Cambridge, MA: National Bureau of Economic Research; 2003. (NBER Working Paper,9732).

9. Beyer H. Educación y desigualdad de ingresos: una nueva mirada. Estud Públicos.2000;(77): 97-130.

10. Beyer H. Productividad, desigualdad y capital humano: Los complejos desafios de Chile. Estud Públicos.2005;(97):59-98.

11. Beyer H. ¿Qué Veinte Años No Es Nada...? Una Mirada a la Desigualdad de Ingresos a partir de las Encuestas CASEN.Estud Públicos.2011;(121):5-33.

12. Sapelli $C$. Ecuaciones de Mincer y las tasas de retorno a la educación en Chile: 1990-1998. Santiago: Pontificia Universidad Católica de Chile; 2003.

13. Sapelli C. Returns to Schooling and Income Distribution by Cohort in Chile:
An Analysis Based on Synthetic Panel Data. Santiago: Pontificia Universidad Católica de Chile; 2005. (Documentos de Trabajo, 290).

14. Antunezlrgoin $C$. Análisis de series de tiempo. Contribuciones a la Economía. $2011 ;(02): 1-47$.

15. Contreras D, Melo E, Ojeda S.¿Estimando el retorno a la educación o a los no observables?: Evidencia de datos de Panel. Estud Econ. 2005;32(2):187-199.

16. Mizala A, Romaguera P. Remuneraciones y tasas de retorno de los profesionales chilenos. En: Bruner JJ, Meller P, editores. Oferta y demanda de profesionales y técnicos en Chile: El rol de la información pública.Santiago: RIL; 2003. p. 171-209.

17. Chile. Ministerio de Desarrollo Social. Encuesta CASEN 2011. Encuesta de Caracterización Socioeconómica Nacional [en línea]. Santiago: Ministerio de Desarrollo Social; 2012 [citado mar 2015]. Disponible en: http://observatorio. ministeriodesarrollosocial.gob.cl/layout/doc/casen/publicaciones/2011/ CASEN_2011.pdf

18. Collins R. La sociedad credencialista. Sociología históricade la educación y de la estratificación. Madrid: AKAL; 1989.

19. Riveros L. El Retorno Privado y Social de la Educación en Chile. Cuad Econ.1990; 60:191-210.

20. Merlo J. Retornos a la Educación durante una Depresión Económica. Evidencia Empírica para Argentina [tesis]. Santiago: Pontificia Universidad Católica de Chile; 2009.

21. Urzúa, S. La Rentabilidad de la Educación Superior en Chile. Estudios Públicos. 2012;(386):1-43.

22. Uribe J, Castellar C. Una Aproximación Econométrica a la Tasa de Retorno Social de la Educación. Rev Soc Econ.2001;1:77-102. 